\title{
Sustancias dopantes y su incidencia: una visión retrospectiva del laboratorio nacional de prevención y control del dopaje de México
}

\section{Banned substances and their incidence: A retrospective view of the national laboratory of prevention and doping control of Mexico}

\author{
Karina Mercado Soberanes*, Evangelina Camacho Frías*, Leonardo Rodríguez Balandrán*, \\ Martha Elena Rodríguez Fermán*, Nancy Mendoza Méndez*; Benjamín Velasco-Bejarano*, **. \\ * Laboratorio Nacional de Prevención y Control del Dopaje, Comisión Nacional de Cultura Física y Deporte, Camino Sta. \\ Teresa \# 482, Col. Peña Pobre Delegación Tlalpan, Ciudad de México, C.P. 14060, México. ** Sección de Química Orgánica, \\ Departamento de Ciencias Químicas Facultad de Estudios Superiores Cuautitlán, Universidad Nacional Autónoma de México, \\ Av. 1 de Mayo, S/N, Col. Sta. Ma. Las Torres, Cuautitlán Izcalli, Estado de México, C.P. 54740, México.
}

\section{Resumen}

El uso de sustancias para incrementar el desarrollo deportivo de atletas ha sido un flagelo en el deporte internacional. En este sentido la Agencia Mundial Antidopaje (WADA-AMA) ha implementado una serie de estándares que permiten armonizar la lucha contra el dopaje desde diferentes aristas. Particularmente los laboratorios acreditados por la WADA-AMA forman parte importante en la erradicación de dopaje deportivo. En este informe se muestran los datos obtenidos en el Laboratorio Nacional de Prevención y Control del Dopaje (LNPCD-CONADE) de acuerdo a la incidencia de Resultados Analíticos Adversos (RAA) en el periodo 2009-2015, los cuales fueron obtenidos del análisis de un total de 18,085 muestras biológicas de control antidopaje. Se hace un análisis de la distribución de muestras de acuerdo al género, tipo de deporte ya sea en competición o fuera de competición, así como de la prevalencia de RAA durante el periodo de tiempo analizado y la relación respecto al grupo de sustancia dopante y tipo de deporte. Los datos aquí presentados se compararon con los disponibles en la página electrónica de la WADA-AMA y se observó que en los casos de sustancias del grupo S1 es más alto el porcentaje que se reporta por el LNPCD-CONADE que el reportado a nivel mundial, caso contrario se determinó para RAA que presentaron alguna sustancia del grupo S6 y S8. Así mismo se observa una mayor prevalencia en el uso de sustancias dopantes por atletas masculinos (75\%) comparado con el $25 \%$ observado en atletas femeninos. Los deportes con mayor número de RAA detectados en el laboratorio fueron béisbol, ciclismo y atletismo

Palabras clave: Control antidopaje; Sustancias dopantes; Laboratorio Nacional de Prevención y Control del Dopaje-CONADE; ISO/IEC17025 .

\section{Abstract}

The use of banned substances to increase athletes' performance has been a scourge in international sport. In this sense, the World AntiDoping Agency (WADA-AMA) has implemented a series of standards that harmonize the fight against doping. In particular, accredited WADA-AMA laboratories play an important role in the eradication of sports doping. This report shows the data obtained in the National Laboratory of Prevention and Control of Doping (LNPCD-CONADE) according to the incidence of Adverse Analytical Findings (AAF) in the 2009-2015 interval, which were obtained from the analysis of a total of 18,085 biological doping-control samples. The distribution of samples was analyzed as a function of gender, type of sport either in competition or out of competition, as well as the prevalence of AAF during the period of time analyzed and the relation regarding group of doping substance and type of sport. The data presented here were compared with those reported worldwide by the WADA-AMA and it was observed that in the cases of substances of the S1 group, the percentage reported by the LNPCD-CONADE is higher than the one reported worldwide. The opposite was observed for AAF presented by some substance from Groups S6 and S8. Likewise, a higher prevalence in the use of doping substances by male athletes $(75 \%)$ is observed compared to that observed in female athletes $(25 \%)$. The sports with the highest number of AAF detected in the laboratory were baseball, cycling, and athletics.

Keywords: Anti-Doping Control; Banned substances; National Antidoping Laboratory-CONADE; ISO/IEC-17025. 
$\mathrm{E}$ 1 dopaje deportivo es considerado como el uso de alguna sustancia o método físico que permite el incremento de la capacidad física de un atleta de manera artificial, en este sentido, la Agencia Mundial Antidopaje (WADA-AMA: World Anti-Doping Agency) fue creada a finales del 1999 con la finalidad de organizar y gestionar los esfuerzos encaminados al no uso de sustancias dopantes en el deporte. Desde sus inicios esta agencia publicó un documento denominado estándar internacional para laboratorios (EIL) (WADA-AMA, ISL, 2009, 2012, 2015) para armonizar el análisis de muestras biológicas de control antidopaje en sus laboratorios acreditados. Varios países antes de la creación de la WADA-AMA, ya contaban con un laboratorio reconocido por la comisión médica del Comité Olímpico Internacional (COI) para realizar este tipo de análisis, posteriormente, estos pasaron a ser acreditados bajo el estándar internacional para laboratorios emitido por la WADA-AMA. Hemmersbach (2008) presenta algunos datos históricos de los laboratorios antidopaje hasta nuestros días. Así mismo otros autores han descrito el impacto y las implicaciones que tiene el dopaje deportivo en la sociedad y en la comunidad deportiva (Ramos, 1999; Catlin, Fitch y Ljungqvist, 2008; Dvorak, Saugy y Pitsiladis, 2014; Atienza, López y Pérez, 2014; Smith y Stewart, 2015). México, para ese momento contaba con un laboratorio destinado a cubrir las necesidades de análisis de muestras biológicas de control antidopaje de atletas mexicanos, por lo que, la Comisión Nacional de Cultura Física y Deporte (CONADE), organismo rector de las políticas deportivas en México, dirigió sus esfuerzos para obtener la acreditación internacional de su laboratorio bajo el estándar de la WADA-AMA (WADA-AMA, ISL, 2009, 2012, 2015). En este sentido México, se unió a la convención internacional contra el uso de sustancias dopantes en el deporte, promovida por la UNESCO y en el año 2007, reafirma su compromiso con esta causa al ratificarla por medio de un decreto publicado en el Diario Oficial de la Federación en el mismo año (DOF 2007), además de apoyar de manera contundente la acreditación de su laboratorio.

En el año 2009 el Laboratorio Nacional de Prevención y Control del Dopaje (LNPCD-CONADE) de México, mediante una colaboración con el Catalonian Antidoping Laboratory Fundació Institut Mar D’Investigacions Mèdiques (IMIM) de Barcelona España, laboratorio acreditado por la WADA-AMA, comenzaron a implementar de manera organizada un sistema de gestión de la calidad basado en la Norma ISO/EC-17025, requisito indispensable para obtener la acreditación de la WADA-AMA, para ese momento ya se habían iniciado los preparativos para la celebración de los XVI Juegos Panamericanos 2011 que se realizarían en el mes de octubre en la ciudad de Guadalajara Jalisco, México. Por lo que, el LNPCD-CONADE junto con el Catalonian Antidoping Laboratory, se encargaron del análisis de las muestras biológicas de control de dopaje de este importante evento bajo la modalidad de laboratorio satélite contemplado en el estándar internacional para laboratorios (WADA-AMA, ISL, 2009, 2012, 2015).

Posteriormente en noviembre del 2012, el LNPCD-CONADE obtuvo la acreditación de su sistema de gestión de la calidad bajo la Norma NMX-EC-17025-IMNC-2006 (IMNC, 2006), por parte de la Entidad Mexicana de Acreditación, A.C. (EMA), y finalmente en junio del 2013 y después de aprobar todos los exámenes técnicos, de contar con un organigrama acorde a los estándares internacionales y de cumplir con el total de los requisitos exigidos por la WADA-AMA, se recibió la acreditación internacional. Por lo que, el objetivo de este estudio descriptivo es dar a conocer la incidencia del uso de sustancias dopantes en atletas a partir de los resultados generados del análisis de las muestras biológicas de control antidopaje entregados al LNPCD-CONADE durante el periodo 2009 a 2015. No se presentan los nombres de los atletas dado que no es un dato que deba conocer el laboratorio además de que no se incluye en los formatos de control antidopaje que se entregan para su análisis. Sólo se hará referencia al tipo deporte (olímpico u no olímpico), el género, además de la sustancia dopante detectada de acuerdo a la clasificación que para tal caso hace la WADA-AMA (WADA-AMA, PL, 2009, 2010, 2011, 2012, 2013, 2014, 2015), datos que son comparados con la información disponible en el portal electrónico de la WADA-AMA en el mismo periodo de tiempo (WADA-AMA, WLS, 2009, 2011, 2012, 2013, 2014, 2015). Es importante mencionar que la selección de controles antidopaje es aleatoria por lo que no todo el universo de atletas está incluido en todos los años para su evaluación.

\section{Métodos}

En este estudio, se consideraron únicamente los resultados obtenidos del análisis de un total de 18,085 muestras biológicas de orina, las cuales fueron recibidas durante el periodo comprendido entre los años 2009 a 2015. Estas muestras de acuerdo al EIL publicado por la WADA-AMA (WADA-AMA, ISL, 2009, 2012, 2015), están clasificadas como muestras en competición (MEC) y como muestras fuera de competición (MFDC) (WADA-AMA, SCP 2006).

Las muestras biológicas de orina fueron recolectadas por oficiales de control de dopaje (OCD) que es personal externo al laboratorio, los cuales se encargan de la recolección, traslado y custodia de las mismas hasta su entrega en el laboratorio como lo indica la WADA-AMA en su documento SCP. (WADA-AMA, SCP, 2006) Estas muestras fueron recolectadas durante todo el año y no son dependientes del periodo estacional, así como tampoco del género, edad, deporte o federación deportiva.

Todas las muestras se recibieron y registraron para su distribución y análisis inicial de tamizaje empleando 
los métodos analíticos internos de operación, los cuales están validados de acuerdo a las normas internacionales aplicables, así como a la información científica disponible en su momento (Arnedo, Ricarte, Martínez y Salvador, 1998; Mareck, Geyer, Opfermann, Thevis y Schänzer, 2008; Kickman y Cowan, 2009; Sottas, Robinson, Rabin y Saugy, 2011; Botrè, De la Torre y Mazzarino, 2016). Además se siguieron las indicaciones que se mencionan en los diferentes documentos técnicos y guías emitidas por la WADA-AMA, (WADA-AMA, TD-DL, 2010, 2012, 2013, 2014; WADA-AMA, EAAS, 2004-2014; WADA-AMA, IRMS, 2014; WADA-AMA, IDCR, 2010, 2013, 2015; WADA-AMA, TD-MRPL, 2009, 2010, 2012, 2014, 2015; WADA-AMA, NAND, 2004; WADA-AMA, TD-EPO, 2009, 2013, 2014; WADA-AMA, LDOC, 2009; WADA-AMA, ICOC, 2009) con la finalidad de detectar las sustancias dopantes o sus metabolitos o sus marcadores descritos en la lista de sustancias y métodos prohibidos que para tal caso la WADA-AMA publica al inicio de cada año. (WADA-AMA, PL, 2009, 2010, 2011, 2012, 2013, 2014, 2015) El análisis inicial de tamizaje para la detección de hormona luteinizante (LH) por sus siglas en inglés y la gonadotrofina coriónica (hCG) por sus siglas en inglés se realizó empleando los equipos analíticos de inmunoensayo Axym, y COBAS e411. La confirmación de una muestra sospechosa esto es, que en el tamizaje inicial se detectó la posible presencia de una sustancia dopante o sus metabolitos o sus marcadores de acuerdo a la lista antes mencionada, se realizó empleando métodos analíticos específicos desarrollados para cada una de ellas.

De acuerdo a los documentos técnicos de la WADA-AMA, las sustancias clasificadas con un umbral de corte, (WADA-AMA, TD-DL, 2010, 2012,2013, 2014) fueron cuantificadas y confirmadas previo a la emisión del resultado analítico. El resto de las sustancias que no se incluyen en esta categoría, su sola presencia a un límite de detección aceptable en una muestra de orina constituye un RAA. Todas las muestras independientemente de su resultado fueron informadas al correspondiente organismo solicitante, mediante el procedimiento implementado por el laboratorio y desde el año 2013 se informan a través del sistema de administración y gestión de resultados ADAMS por sus siglas en inglés de la WADA-AMA.

\section{Resultados}

En la Tabla 1 se presenta una descripción del equipamiento analítico con que cuenta actualmente el LNPCD-CONADE, así como el grupo de sustancias dopantes que se detectan en cada caso, de acuerdo a la lista de sustancias y métodos prohibidos emitido por la WADA-AMA (se incluyen las sustancias padre, sus metabolitos o marcadores). Este equipamiento en su mayoría fue adquirido durante el periodo de tiempo aquí analizado.
Tabla 1. Equipamiento analítico del LNPCD-CONADE.

\begin{tabular}{|c|c|c|}
\hline Equipo analítico & $\begin{array}{l}\text { Número de } \\
\text { instrumentos }\end{array}$ & Grupo de sustancia detectada \\
\hline CG-EM (GC-MS) & 10 & $\mathrm{~S} 1, \mathrm{~s} 3, \mathrm{S6}, \mathrm{S7}, \mathrm{S} 8$ \\
\hline CG-DNF (GC-NPD) & 3 & S6, S7 \\
\hline CL-ES (LC-MS) & 7 & $\mathrm{~S} 1, \mathrm{~S} 3, \mathrm{~S} 4, \mathrm{~S} 5, \mathrm{~S} 6, \mathrm{S7}, \mathrm{S} 9, \mathrm{P} 2$ \\
\hline CLAP-ADD (HPLC-DAD) & 1 & S1 \\
\hline CG-C-EMRI (GC-C-IRMS) & 2 & S1 \\
\hline CG-EM $3 Q$ (GC-MS 3Q) & 2 & S1 \\
\hline Luminómetro de tubos & 1 & S2 \\
\hline Citómetro de flujo & 1 & S2 \\
\hline $\begin{array}{l}\text { Inmunoanálisis } \\
\text { (IMMULITE 1000, } \\
\text { COBAS e411, Advia } \\
\text { Centaur XP) }\end{array}$ & 3 & S2 \\
\hline
\end{tabular}

Nota. * ${ }^{\mathrm{S}} 1$ : Agentes anabolizantes; $\mathrm{S} 2$ : Hormonas peptídicas, factores de crecimiento, sustancias afines y miméticos; S3: $\beta-2$ agonistas; $S_{4}$ : Moduladores hormonales y metabólicos; S5: Diuréticos y agentes enmascarantes; S6: Estimulantes; S7: Narcóticos; S8: Cannabinoides; S9: Glucocorticoesteroides; $\mathrm{P}_{2}$ : $\beta$-bloqueadores.

Como se mencionó previamente, 18,085 resultados analíticos de muestras de orina se incluyeron en este estudio, en la Figura 1 se muestra la distribución del número de muestras recibidas por año en el periodo 2009 a 2015. Se analizaron orinas provenientes de 48 diferentes disciplinas deportivas clasificadas como olímpicas y no olímpicas, en este sentido, es conveniente mencionar que sólo hasta el año 2015 se comenzó a recibir muestras de sangre, datos que no se incluyen en este estudio, aunque ya se habían analizado algunas solicitadas por el comité organizador de los XXII Juegos Deportivos Centroamericanos y del Caribe en el año 2014 en donde el LNPCD-CONADE realizó el análisis de las muestras generadas en este evento. En la misma figura se observa que a partir del año en que se otorgó la acreditación por parte de la WADA-AMA, el número de muestras recibidas se incrementó sustancialmente y de manera continua respecto a los años previos.

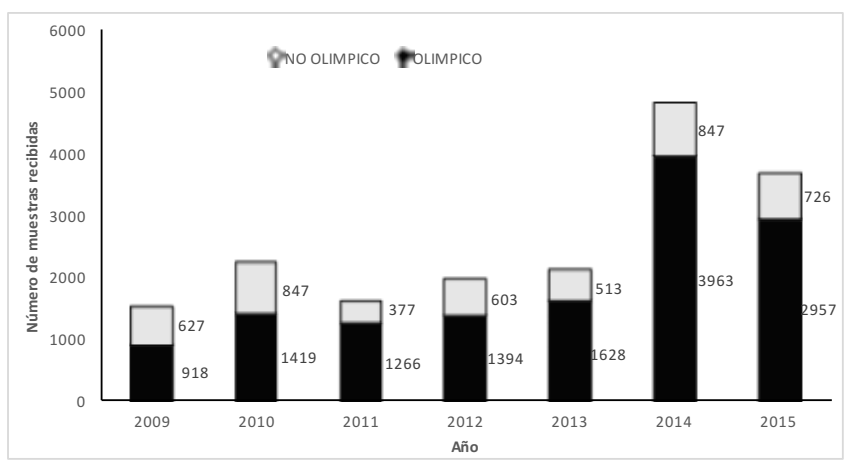

Figura 1. Total de muestras de orina recibidas durante el periodo 2009-2015, organizadas por tipo de deporte.

En este sentido en la Figura 2, se muestra el total de RAA reportados por año por el LNPCD-CONADE, independientemente del grupo de sustancias detectada, tanto 
para deportes olímpicos como no olímpicos. Es conveniente mencionar que el porcentaje de RAA fue mayor en los deportes no olímpicos en la mayoría de los años que se incluyeron en este estudio entre los deportes no olímpicos se encuentran futbol americano, powerlifting, fisicoculturismo, Ju-Jitsu, entre otros.

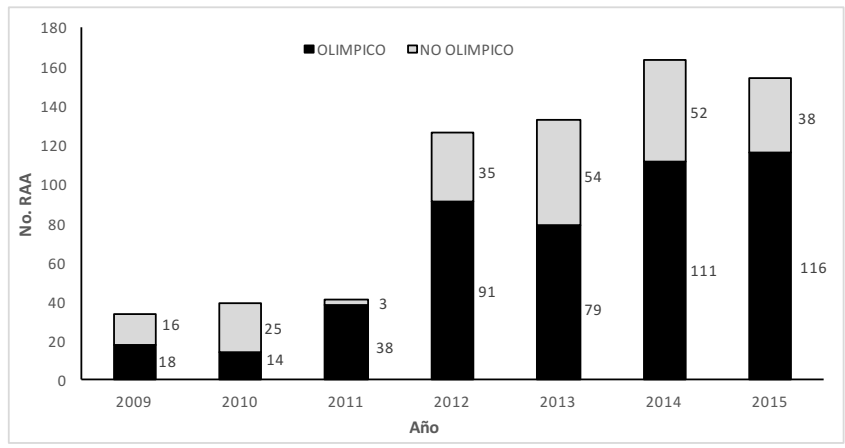

Figura 2. Total de RAA informados durante el periodo 2009-2015 para deportes olímpicos y no olímpicos.

En el caso del total de RAA distribuidos por género los cuales se presentan en la Figura 3, se observa que es mayor la incidencia en el género masculino que en el femenino, en este caso no se presenta una distribución por tipo de sustancia relacionada con el género del deportista.

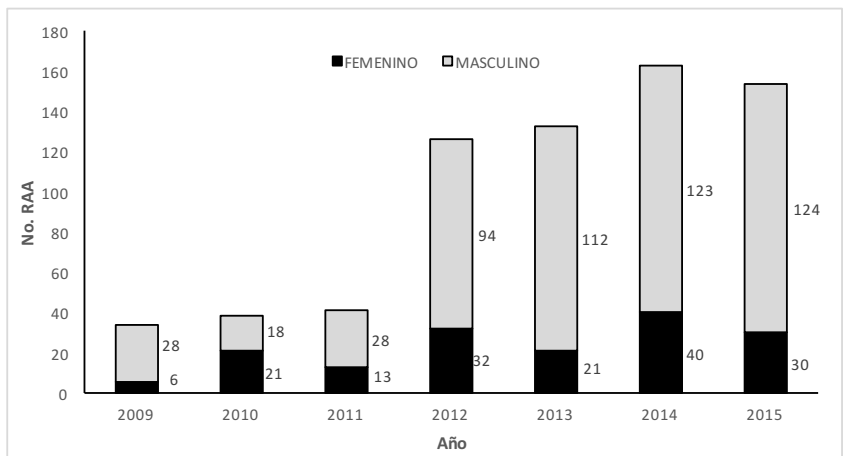

Figura 3. Total de RAA informados durante el periodo 2009-2015 distribuido por género masculino y femenino.

En la Tabla 2, se presenta el porcentaje total de RAA por año reportados por el LNPCD-CONADE así como el porcentaje anual de RAA informado a nivel mundial en su página electrónica por la WADA-AMA. Al respecto se observa que, el porcentaje reportado por el laboratorio mexicano en cada año está por arriba del valor porcentual informado por la WADA-AMA.

Del análisis en conjunto de las 18,085 muestras incluidas, se determinó que, el porcentaje de RAA reportados por el LNPCD-CONADE fue de $3.8 \%$. En este sentido y para detallar de una manera más precisa esta información, en la Tabla 3 se presentan los datos organizados por año relacionados con el grupo de la sustancia detectada. Es im-
Tabla 2. \% de resultados analíticos adversos informados por el LNPCD-CONADE durante el periodo 2009-2015

\begin{tabular}{cccccccc}
\hline Tipo de deporte & $\mathbf{2 0 0 9}$ & $\mathbf{2 0 1 0}$ & $\mathbf{2 0 1 1}$ & $\mathbf{2 0 1 2}$ & $\mathbf{2 0 1 3}$ & $\mathbf{2 0 1 4}$ & $\mathbf{2 0 1 5}$ \\
\hline Olímpico & 2.0 & 1.0 & 3.0 & 6.5 & 4.9 & 2.8 & 3.9 \\
No Olímpico & 2.6 & 3.0 & 0.80 & 5.8 & 9.0 & 6.1 & 5.2 \\
WADA-AMA $^{*}$ & 0.90 & 0.90 & 1.0 & 0.99 & 0.97 & 0.77 & 0.83 \\
\hline
\end{tabular}

Nota. *Porcentaje de RAA, informados por la WADA-AMA en su página electrónica, sólo para deportes olímpicos

Tabla 3. Distribución de RAA por grupo de sustancias dopantes, durante el periodo 2009-2015

\begin{tabular}{ccccccccc}
\hline Grupo & $\mathbf{2 0 0 9}$ & $\mathbf{2 0 1 0}$ & $\mathbf{2 0 1 1}$ & $\mathbf{2 0 1 2}$ & $\mathbf{2 0 1 3}$ & $\mathbf{2 0 1 4}$ & $\mathbf{2 0 1 5}$ & $\begin{array}{c}\text { Total } \\
\text { acumulado } \\
\text { n (\%) }\end{array}$ \\
\hline S1 & 18 & 15 & 30 & 90 & 109 & 132 & 141 & $535(75.54 \%)$ \\
S2 & - & - & - & - & - & 1 & - & $1(0.14 \%)$ \\
S3 & 1 & - & - & - & - & - & - & $1(0.14 \%)$ \\
S4 & - & - & - & - & - & - & 1 & $1(0.14 \%)$ \\
S5 & 2 & 13 & 2 & 5 & 3 & 13 & 4 & $42(6.09 \%)$ \\
S6 & 6 & 6 & 7 & 17 & 17 & 9 & 4 & $66(9.57 \%)$ \\
S7 & - & 1 & - & - & - & 1 & - & $2(0.29 \%)$ \\
S8 & 6 & 3 & - & 12 & 2 & - & - & $23(3.33 \%)$ \\
S9 & 1 & 1 & 2 & 2 & 2 & 6 & 4 & $18(2.61 \%)$ \\
P2 & - & - & - & - & - & 1 & - & $1(0.14 \%)$ \\
TOTAL & 34 & 39 & 41 & 126 & 133 & 163 & 154 & 690 \\
POR AÑO & & & & & & & & \\
\hline
\end{tabular}

portante hacer notar que sólo a partir de junio del 2013, los resultados emitidos por el laboratorio, tuvieron validez internacional, anterior a esta fecha sólo eran reconocidos por el Comité Nacional Antidopaje de México y la federación deportiva mexicana involucrada.

Cuatro fueron los grupos de sustancias dopantes que de acuerdo a la lista de sustancias y métodos prohibidos emitida por la WADA-AMA, presentaron la mayor incidencia y que corresponden a los grupos: S1 Agentes anabolizantes (535 RAA), S5 Diuréticos (42 RAA), S6 Estimulantes (66 RAA) y S8 Cannabinoides (23 RAA), cada uno de ellos se discutirán de manera detallada en este documento.

\section{Agentes anabolizantes (Grupo S1)}

La presencia de sustancias incluidas dentro del grupo S1 (WADA-AMA, WLS, 2009, 2011, 2012, 2013, 2014, 2015), fueron las más frecuentemente detectadas por el LNPCD-CONADE durante el periodo de tiempo aquí analizado. En la Figura 4 se muestra el porcentaje de RAA de este grupo reportados por el LNPCD-CONADE, durante el periodo 2009-2015 comparados con el total de RAA informados a nivel mundial por la WADA-AMA. En particular, en año 2015 el 92\% de todos los RAA que el LNPCD-CONADE informo a sus clientes, corresponde a sustancias incluidas en este grupo de las cuales ya varios autores ha- 
bían publicado métodos analíticos para su identificación (Donike, 2011; Delgadillo, et al., 2012; Saugy, Lundby y Robinson, 2014; Thevis, Kuuranne, Geyer y Schänzer, 2017; Avella y Medellín, 2012). Esto muestra que los agentes anabólicos siguen siendo las sustancias que con mayor incidencia usan los atletas para incrementar su desarrollo deportivo por lo menos en el universo de resultados aquí estudiados. Este tipo de sustancias se emplea para incrementar la masa muscular y por ende la fuerza, usualmente se detectan por cromatografía de gases acoplado a espectrometría de masas previa derivatización de la molécula. Recientes estudios realizados por González-Martí en 2017 (González-Martí, Fernández-Bustos, Contreras y Sokolova, 2018) en una población española de atletas fisicoculturistas y levantadores de pesas que presentan dismorfia muscular, se demostró que por lo menos el $50 \%$ de ellos consumen esteroides anabólicos androgénicos para tratar este problema.

De los 535 RAA, el $11.03 \%$ correspondió a la presencia

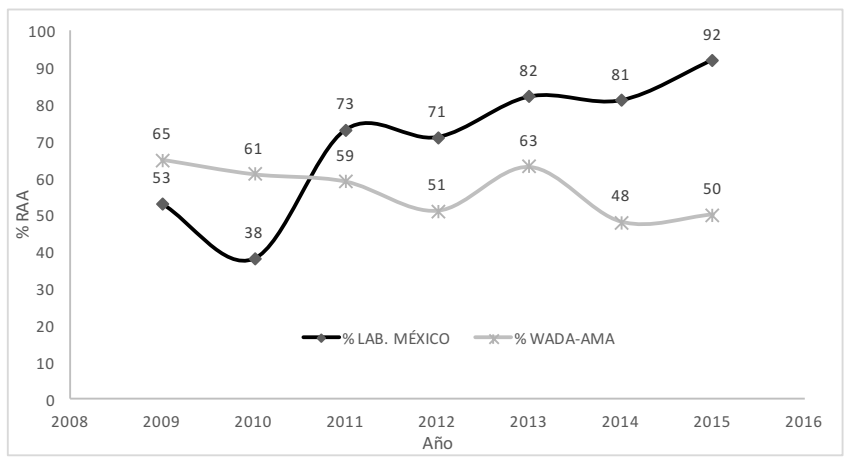

Figura $4 . \%$ de RAA informados por el LNPCD-CONADE durante el periodo 2009-2015 por la presencia de sustancias del grupo $\mathrm{S}_{1}$ Agentes Anabolizantes, vs \% de RAA reportados por la WADAAMA en el mismo periodo.

de Nandrolona, la Boldenona se detectó en un 2.62\%, el Estanozolol y sus metabolitos se identificó en un $4.67 \%$ de las muestras, Clenbuterol se detectó en un $77.57 \%$. Etiocolanolona, Epimetendiol, Drostanolona, Metandrolona, Danazol, Androsterona, Nandrosterona, Metandienona, Metiltestosterona, Oxandrolona, Gestrinona, Epitrenbolona, Mesterolona, Metenolona constituyeron todas juntas el $4.11 \%$ de los RAA.

Si se observa con detalle la Figura 4 se puede identificar que los porcentajes de RAA por sustancias del grupo S1 reportados por el LNPCD-CONADE son más altos con respecto a los informados por la WADA-AMA.

\section{Diuréticos y agentes enmascarantes (Grupo S5)}

En la Figura 5, se muestra el porcentaje de RAA, debidos a la presencia de sustancias del Grupo S5 diuréticos y agentes enmascarantes, según la clasificación que para tal caso realiza la WADA-AMA (WADA-AMA, WLS, 2009,
2011, 2012, 2013, 2014, 2015). Varios estudios reportados en la literatura científica (Cadwallader, De la Torre, Tieri y Botrè, 2010; Thörngren, Östrvall y Garle, 2008; Koehler et al., 2011), hacen referencia a los métodos analíticos para la detección de esta familia de sustancias.

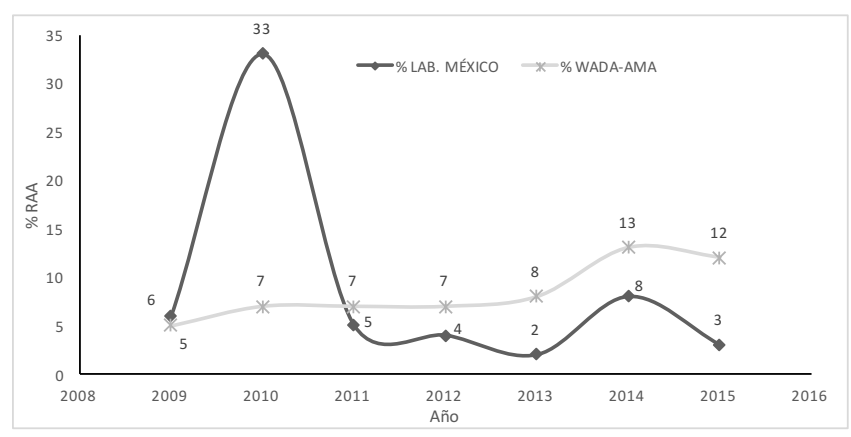

Figura 5. \% de RAA informados por el LNPCD-CONADE durante el periodo 2009-2015 por la presencia de sustancias del grupo S5 Diuréticos, vs \% de RAA reportados por la WADA-AMA en el mismo periodo.

De manera general la tendencia de RAA por diuréticos reportados por el LNPCD-CONADE se mantuvo por debajo de la tendencia mundial. Esto no ocurrió particularmente en el año 2010 en donde fue mayor el porcentaje interno reportado por el laboratorio $33 \%$ con respecto a lo reportado por la WADA-AMA en ese mismo año. De manera general, ésta familia de sustancias son detectadas empleando cromatografía de líquidos acoplada a espectrometría de masas. Específicamente los diuréticos incrementan la diuresis según lo descrito por Brunton, 2012 (Brunton, Chabner y Knollman, 2012), por lo que suelen ser usados para favorecer la rápida eliminación de sustancias exógenas presentes en el cuerpo humano. No resulta sorprendente detectar este tipo de fármacos en el laboratorio en combinación con algún otro tipo de sustancia dopante, lo que se considera dopaje deportivo. Los porcentajes de estas sustancias que se detectaron en el periodo de tiempo estudiado son los siguientes: Furosemida 47.62\%, Clortalidona 19.05\%, Hidroclorotiazida 30.95\%, Bumetanida $2.38 \%$.

\section{Estimulantes (Grupo S6)}

La detección de sustancias dopantes del grupo S6 (WADA-AMA, WLS, 2009, 2011, 2012, 2013, 2014, 2015) que corresponde a los estimulantes, sólo es requerida en MEC, esto debido a que el efecto farmacológico mostrado es inmediato y a corto plazo, según lo que se indica en la literatura consultada (Brunton, Chabner y Knollman, 2012) por lo que, no se considera relevante su detección cuando están fuera de competición. Varios autores han reportado interesantes propuestas analíticas para la detección apropiada de este tipo de sustancias en muestras biológicas 
de orina, empleando la técnica de cromatografía líquida acoplada a espectrometría de masas (Deventer et al., 2009; Barroso et al., 2012; Beuck et al., 2012; Monfort, Martínez, Bergés, Segura y Ventura, 2015; O’Byrne, Kavanagh, McNamara y Stokes, 2013; Marclay, Grata, Perrenoud y Saugy, 2011; Strano, Abate, Bragano y Botrè, 2009).

En la Figura 6 se muestra el porcentaje de RAA en muestras de atletas mexicanos debido a la presencia de alguna sustancia de este grupo. Es importante hacer notar el número de RAA por sustancias del grupo S6, disminuyó en los últimos años hasta un $3 \%$ en comparación con el total de los RAA disponibles en la página de la WADA-AMA, que por el contrario se incrementaron hasta un $15 \%$ en el año 2015. $21 \%$ de RAA atribuidos a la presencia de estimulantes corresponde a muestras de atletas en competencia, la mayor incidencia se observa en deportes no olímpicos. Las sustancias del grupo S6 con mayor incidencia fueron: Anfetamina $(39.39 \%)$, Pseudoefedrina $(4.55 \%)$, Metilhexaneamina $(37.88 \%)$, Cocaína $(7.58 \%)$, Oxilofrina $(3.03 \%)$, el porcentaje restante esta distribuido entre Metilfenidato, Isometepteno, Fentermina, Octopamina que en algunos casos no revasa el 1.5\% de manera individual. Es importante aclarar que los porcentajes de RAA que se muestran por el LNPCD-CONADE, son calculados a partir del total de resultados reportados por año.

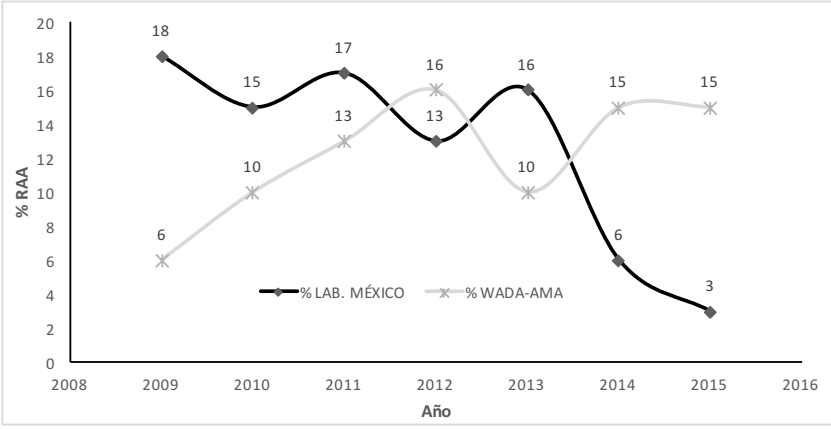

Figura 6. \% de RAA informados por el LNPCD-CONADE durante el periodo 2009-2015 por la presencia de sustancias del grupo S6 Estimulantes, vs \% de RAA reportados por la WADA-AMA en el mismo periodo.

\section{Cannabinoides (Grupo S8)}

El último de los cuatro grupos que se consideró con una mayor incidencia de RAA fue el S8 (WADA-AMA, WLS, 2009, 2011, 2012, 2013, 2014, 2015) debido a la presencia de cannabinoides como los descritos por autores como Mareck et al. (2009), Castaneto et al. (2015), Möller et al. (2011), Chebbah, Pozo, Deventer, Van Eenoo y Delbeke, (2010) que describen métodos analíticos para la detección y confirmación de la presencia de este tipo de sustancias en muestras de orina. En la Figura 7 se describe el porcentaje de RAA debidos a la presencia de este tipo de sustancias comparado con los datos reportados por la WADA-AMA en un periodo similar de tiempo.

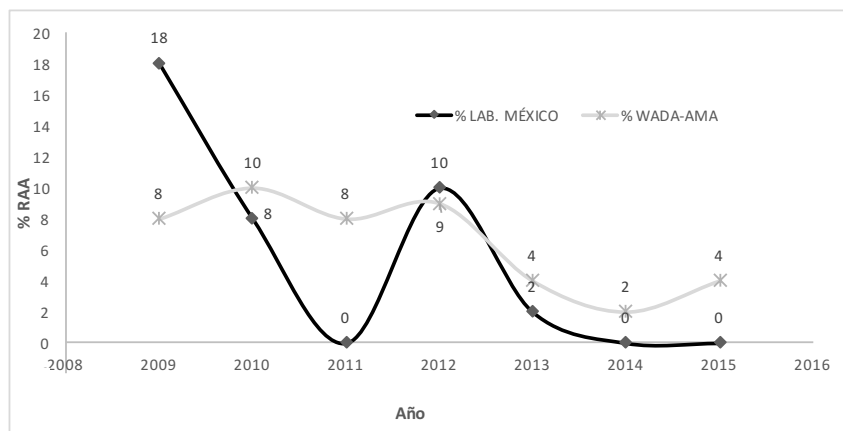

Figura $7 . \%$ de RAA informados por el LNPCD-CONADE durante el periodo 2009-2015 por la presencia de sustancias del grupo S8 Cannabinoides, vs \% de RAA reportados por la WADA-AMA en el mismo periodo.

Es conveniente mencionar que hasta el año 2013, se consideraba una muestra con un RAA a aquellas que presentaban una concentración de THC y/o sus metabolitos por arriba de $15 \mathrm{ng} / \mathrm{mL}$. Posteriormente a mediados del 2014 este valor fue modificado y se incrementó hasta un límite de decisión de $180 \mathrm{ng} / \mathrm{mL}$ con una incertidumbre del 10\%; por lo que el número de RAA debido a la detección de cannabinoides muestra una disminución en el LNPCD-CONADE hasta llegar a cero, tendencia que se observó hasta el año 2015. Este tipo de sustancia se detecta regularmente por cromatografía de gases acoplado a espectrometría de masas.

\section{Discusión}

Posterior a la obtención de la acreditación internacional del LNPCD-CONADE otorgado por la WADA-AMA en el año 2013, se incrementó el número de muestras biológicas que se recibieron en este laboratorio ya no solamente de federaciones mexicanas que enviaron sus muestras, si no que ahora también de federaciones y organismos deportivos internacionales tales como la UCI, IAAF, FIFA, FINA, CONMEBOL, etc.

Si bien, los métodos de análisis empleados desde el año 2009 hasta el año 2011 no se encontraban acreditados por la Norma ISO/IEC-17025, estos métodos se habían desarrollado y posteriormente validado siguiendo un riguroso proceso acorde a los documentos técnicos disponibles y a las normas técnicas internacionales aplicables para métodos analíticos, por lo que, los resultados obtenidos mediante estas metodologías son válidos y pueden ser comparados con los disponibles en el portal electrónico de la WADA-AMA (Kioukia et al., 2014; Aguilar et al., 2017). Es conveniente mencionar que el tipo de metodología analítica para la detección de la mayoría de las sustancias, son de libre elección por los laboratorios siempre y cuando se alcancen los niveles de detección y cuantificación exigidos por la WADA-AMA. Otros métodos como por ejemplo los empleados para determinar el origen de sustancias endó- 
genas que pudieron haber sido consumidas de manera exógena, cuentan con una especificación precisa del tipo de equipamiento y metodología a emplear la cual es la espectrometría de masas de relaciones isotópicas (IRMS), así como un documento técnico que indica los pasos a seguir tanto en la preparación de la muestra, el método de análisis y la manera de reportar los resultados obtenidos (WADA-AMA, TD-IRMS, 2014). El porcentaje total de RAA reportados en el periodo de tiempo estudiado fue del $3.8 \%$ en este valor se incluyen todos los grupos de sustancias dopantes consideradas por la WADA-AMA en su listado de sustancias y métodos prohibidos.

La recolección de las muestras de orina como se mencionó anteriormente es realizada por personal externo al laboratorio, la toma de muestras no se realizó en un periodo estacional específico, por lo que el tipo de análisis o el tiempo de recolección no son dependientes de la estación del año. La WADA-AMA no ha emitido una consideración respecto al tratamiento que se le deba de aplicar a una muestra recolectada en los diferentes periodos estacionales del año, por lo que, este no es un parámetro que influya o determine el tipo de sustancia dopante detectada. Lo que sí es importante mencionar, es que en el LNPCD-CONADE, se observa un incremento en la recepción de muestras entre los meses de mayo-septiembre, lo anterior posiblemente atribuido a que en este periodo es cuando se realizan un mayor número de competencias deportivas y las federaciones involucradas incrementan la cantidad de controles a sus deportistas, previo a su participación. Aunado a esto, en México por esas fechas se realiza un evento denominado olimpiada nacional, el cual es de carácter local y que sólo involucra a deportistas nacionales, por lo que se envían un buen número de muestras biológicas para su análisis.

Por otro lado, la detección y cuantificación de las sustancias de los diferentes grupos consideradas por la WADA-AMA como dopantes está determinado de acuerdo a los documentos técnicos por ella emitidos. Específicamente los niveles mínimos de rendimiento requeridos, MRPL por sus siglas en inglés "Minimum Required Performance Levels”, es un parámetro analítico obligatorio de desempeño técnico el cual es establecido por WADA-AMA y el laboratorio deberá cumplir cuando confirme la presencia de una sustancia prohibida en particular o su (s) metabolito (s) o marcador (s). Este parámetro se establece para armonizar el rendimiento analítico de los métodos aplicados a la detección de sustancias sin umbral. El MRPL es la concentración mínima de una sustancia prohibida, que los laboratorios deberán ser capaces de detectar e identificar confiablemente en las operaciones diarias de rutina. En particular estos valores de concentración han disminuido a través de los años, tal vez debido a la disponibilidad de equipos analíticos que cuentan con un mayor poder de detección y sensibilidad. Actualmente en el año 2017, los valores del MRPL para cada grupo de sustancias dopantes de acuerdo a los requisitos de la WADA-AMA son los siguientes: $\mathrm{S} 1=5 \mathrm{ng} / \mathrm{mL}, \mathrm{S} 2=2 \mathrm{ng} / \mathrm{mL}, \mathrm{S} 3=20 \mathrm{ng} / \mathrm{mL} \mathrm{S} 4=20$ $\mathrm{ng} / \mathrm{mL}, \mathrm{S} 5=200 \mathrm{ng} / \mathrm{mL}, \mathrm{S} 6=100 \mathrm{ng} / \mathrm{mL}, \mathrm{S} 7=50 \mathrm{ng} / \mathrm{mL}$, $\mathrm{S} 8=1 \mathrm{ng} / \mathrm{mL}, \mathrm{S} 9=30 \mathrm{ng} / \mathrm{mL} \mathrm{P} 2=100 \mathrm{ng} / \mathrm{mL}$. (WADA-AMA, MRPL-2017). Cualquier sustancia que esté incluida en los diferentes grupos y que el laboratorio la detecte a estos niveles, podrá conducir a un resultado analítico adverso. También se puede dar el caso de que un laboratorio pueda detectar alguna de ellas a niveles por debajo del MRPL con la suficiente confiabilidad analítica y podrá reportar una muestra con un resultado analítico adverso.

De acuerdo al total de resultados analíticos adversos reportados por el laboratorio, se observa que, el $75 \%$ de ellos corresponde a muestras obtenidas de atletas masculinos y sólo el 25\% corresponden a atletas femeninos, en ninguno de los grupos de sustancias dopantes se observó que esta relación se invirtiera. De manera más detallada y por grupo de sustancias se observa que para el caso del grupo S1 de un total de 535 RAA el $76 \%$ está asociado a muestras de varones y el $24 \%$ a mujeres. Para el caso de RAA en donde se detectó alguna sustancia del grupo S5 (42 RAA), el 45\% correspondía a muestras de atletas del género femenino y el $55 \%$ correspondieron a atletas del género masculino. Continuando con este análisis se observó que para el caso de la presencia de sustancias estimulantes (grupo S6), de un total de 66 RAA, $80 \%$, esto es, 53 de ellos fueron detectados en muestras de atletas masculinos y sólo 13 muestras $(20 \%)$ correspondían a muestras de mujeres. Por otro lado, en México a partir del año 2008 como se menciona en el Diario Oficial de la Federación del Gobierno de los Estados Unidos Mexicanos (DOF 2008), quedó prohibido el uso de Pseudoefedrina y Efedrina para la fabricación de medicamentos así como se prohibió su importación, por lo que la disminución observada en el número de RAA debido a la presencia de este tipo de sustancias podría verse influenciada por la restricción que se ha puesto para la venta de este tipo de sustancias en el mercado mexicano. En este mismo tenor de un total de 23 RAA del grupo S8, el $91 \%$ de ellos correspondió a muestras recolectadas de hombres y el porcentaje restante fue de mujeres. El resto de los grupos de sustancias respecto al género del atleta no se discuten ya que no representan más del $2 \%$ del total de RAA. Los resultados descritos permiten observar que, el número de RAA debido a todos los grupos de sustancias dopantes es mucho mayor en atletas del género masculino.

Particularmente un dato que es de llamar la atención, es la alta incidencia de RAA por la presencia de Clenbuterol en muestras de orina. El Clenbuterol es una sustancia no esteroidal que a ciertas concentraciones produce efectos androgénicos y ésta se detectó en un $77.57 \%$ de las muestras. Existen antecedentes de una alta probabilidad, de que la presencia de Clenbuterol en una muestra biológica de un atleta pueda ser debida al consumo no intencional de 
carne bovina contaminada con esta sustancia, como lo demuestran varios autores (Guddat, et al., 2012; Thevis et al., 2013), así mismo otros autores han estudiado la relación enantiomérica del Clenbuterol detectada en muestras biológicas de orina, cuando esta sustancia proviene de la ingestión de medicamentos de uso comercial (Thevis, et al., 2013; Parr et al., 2017; Velasco-Bejarano, et al., 2017), independientemente de la información científica disponible es obligatorio que el laboratorio informe de este resultado adverso. El Comité Nacional Antidopaje y la federación deportiva involucrada determinarán si aplican o no una sanción al deportista basado en este resultado analítico. Por otro lado y de manera general los deportes en los que se detectaron una mayor cantidad de RAA fueron béisbol, ciclismo, seguido de atletismo, futbol americano, halterofilia, fisicoculturismo y futbol soccer. En el caso de béisbol las sustancias que más se detectaron fueron las asociadas a los grupos S1, S6 y S8, el mayor número de RAA se observó en el año 2009. En el caso de atletismo y ciclismo la mayoría de los RAA de estos deportes corresponden a la presencia de sustancias del grupo S1 y S6. Para el caso de muestras de orina provenientes de los deportes halterofilia, fisicoculturismo y futbol americano fue constante la presencia de sustancias del grupo S1, y fue casi nula la presencia de sustancias de otros grupos. Es cabe mencionar que de los deportes antes mencionados, el futbol soccer es el que más controles antidopaje realiza, por consiguiente el número de muestras que envía para su análisis es también alto, lo que incrementa la posibilidad de una mayor cantidad de RAA en este caso, podemos mencionar que la mayoría (79\%) de los RAA corresponden a la presencia de Clenbuterol.

Respecto a la presencia de sustancias del grupo S5 en muestras biológicas de control antidopaje es importante mencionar que sólo en los años 2012, 2013 y 2014 se detectaron muestras de orina con la presencia de algún diurético en combinación con alguna sustancia del grupo S1 (3 casos), grupo S6 (1 caso), grupo S8 (1 casos) y grupo S9 (1 caso). El porcentaje reportado por el LNPCD-CONADE en el año 2015 fue más bajo (3\%) comparado con el 12\% reportado a nivel mundial por el resto de los laboratorios acreditados por la WADA-AMA.

De manera general el número de RAA atribuidos a muestras provenientes de deportes olímpicos es el doble de aquellos que fueron obtenidos de deportes no olímpicos. La tendencia en el incremento de RAA en deportes olímpicos puede explicarse debido al mayor número de muestras provenientes de estos deportes que se envían al laboratorio. No fue posible establecer la existencia de una dependencia entre el tipo de sustancia dopante detectada y el género del cual provenga una muestra, sólo se pudo identificar que se observa una mayor incidencia de RAA en el sexo masculino $(75 \%)$ que en el femenino (25\%). En la Figura 8 se muestra la distribución observada por tipo de sustancia dopante respecto al género del deportista.

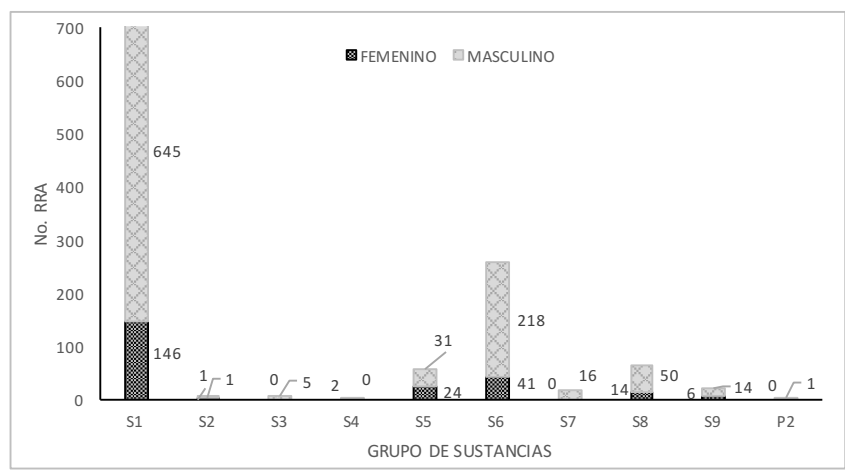

Figura 8. Número de RAA informados por el LNPCD-CONADE durante el periodo 2009-2015 distribuidos por género vs grupo de sustancia dopante.

Sólo en el caso del grupo S5 el número de RAA fue muy cercano entre los dos géneros, en el caso del resto de los grupos de sustancias dopantes no se observó que existiera una mayor prevalencia de RAA para mujeres que para varones. En ese sentido se observó que existe una clara tendencia en la presencia de sustancias de los grupos S1, S6, S7 y S8 en muestras de orina provenientes de deportistas varones.

\section{Reconocimientos}

Se agradece el apoyo y el soporte financiero a la Comisión Nacional de Cultura Física y Deporte (CONADE), asî como a la Secretaria de Educación Pública y a la Secretearía de Hacienda y Crédito Público del Gobierno de los Estados Unidos Mexicanos.

\section{Conflicto de intereses}

Los autores declaran no tener conflicto de intereses.

\section{Referencias}

Aguilar, M., Muñoz-Guerra, J., Plata, M. M. y Del Coso, J. (2017). Thirteen years of the fight against doping in figures. Drug Testing and Analysis, 9, 866-869. doi:10.1002/ dta.2168.

Arnedo, M., Ricarte, J., Martínez, S. y Salvador, A. (1998). Efectos de los esteroides anabolizantes-androgenizantes sobre diversas variables implicadas en el rendimiento deportivo. Revista de Psicología del Deporte, 7, 215-231.

Atienza, E., López, F. y Pérez, L. (2014). El Dopaje y el antidopaje en perspectiva histórica. Materiales para la Historia del Deporte, 12, 94-110.

Avella, R. y Medellín, J. (2012). Los esteroides anabolizantes androgénicos, riesgos y consecuencias. Revista U.D.C.A. Actualidad Ẽ Divulgación Científica, 15 (Supl. Olimpismo), 47-55.

Barroso, O., Godreault, D., Carbó, M., Ayotte, C., Mazzoni, I., Boghosian, T. y Rabin, O. (2012). Determination of 
urinary concentrations of pseudoephedrine and cathine after therapeutic administration of pseudoephedrine-containing medications to healthy subjects: implications for doping control analysis of these stimulants banned in sports. Drug Testing and Analysis, 4, 320-329. doi:10.1002/dta.291.

Beuck, S., Sigmund, G., Kock, A., Schänzer, W., Pokrywka, A., Kiatkowska, D. y Thevis, M. (2012). Identification and characterization of urinary prenylamine metabolites by means of liquid chromatography-tandem mass spectrometry. Drug Testing and Analysis, 4, 701-716. doi:10.1002/dta.1388.

Botrè, F., De la Torre, X. y Mazzarino, M. (2016). Multianalyte LC-MS-based methods in doping control: what are the implications for doping athletes? Bioanalysis, 8, 1129-1132. doi:10.4155/bio-2016-0083.

Brunton, L., Chabner, B. y Knollman, B. (2012). Goodman y Gilman Las Bases Farmacológicas de la Terapéutica. China: MC. Graw Hill.

Cadwallader, A., De la Torre, X., Tieri, A. y Botrè, F. (2010). The abuse of diuretics as performance-enhancing drugs and masking agents in sport doping: pharmacology, toxicology and analysis. British Journal of Pharmacology, 161, 1-16. doi:10.1111/j.1476-5381.2010.00789.x.

Castaneto, M., Scheidweiler, K., Gandhi, A., Wohlfarth, A., Klette, K., Martin, T. y Huestis, M. (2015). Quantitative urine confirmatory testing for synthetic cannabinoids in randomly collected urine specimens. Drug Testing and Analysis, 7, 483-493. doi:10.1002/dta.1709.

Catlin, D., Fitch. K. y Ljungqvist, A. (2008). Medicine and science in the fight against doping in sport. Journal of Internal Medicine, 264, 99-114. doi:10.1111/j.13652796.2008.01993.x.

Chebbah, C., Pozo, O., Deventer, K., Van Eenoo, P. y Delbeke, F. (2010). Direct quantification of 11-nor-D9-tetrahydrocannabinol-9-carboxilic acid in urine by liquid chromatography/tandem mass spectrometry in relation to doping control analysis. Rapid Communications in Mass Spectrometry, 24, 1133-1141. doi:10.1002/rcm.4499.

Delgadillo, M., Garrostas, L., Pozo, O., Ventura, R., Velasco-Bejarano, B., Segura, J. y Marcos, J. (2012). Sensitive and robust method for anabolic agents in human urine by gas chromatography-triple quadrupole mass spectrometry. Journal of Chromatography B, 897, 85-89. doi:10.1016/j.jchromb.2012.03.037.

Deventer, K., Van Eenoo, P., Baele, G., Pozo, O., Van Thuyene, W. y Delbeke, F. (2009). Interpretation of urinary concentrations of pseudoephedrine and its metabolite cathine in relation to doping control. Drug Testing and Analysis, 1, 209-213. doi:10.1002/dta.31.

Donike, M. (2011). The detection of doping by means of chromatographic methods. Drug Testing and Analysis, 3, 15-17. doi:10.1002/dta.238.

DOF, Diario Oficial de la Federación, Órgano del Gobierno Constitucional de los Estados Unidos Mexicanos,
Tomo DCXLV (14), 20 de junio de 2007, primera sección, 5-25.

DOF, Diario Oficial de la Federación, Órgano del Gobierno Constitucional de los Estados Unidos Mexicanos, Tomo DCLVII (10), 13 de junio de 2008, primera sección, 33-35.

Dvorak, J., Saugy, M. y Pitsiladis, Y. (2014). Challenges and threats to implementing the fight against doping in sports. British Journal Sports Medicine, 48, 807-809. doi:10.1136/bjsports-2014-093589.

González-Martí, I., Fernández-Bustos, J. G., Contreras, O. R. y Sokolova, M. (2018). Muscle dysmorphia: detection of the use-abuse of anabolic androgenic steroids in a Spanish sample. Adicciones, 30, 243-250. doi:10.20882/ adicciones.853.

Guddat, S., Fußhöller, H., Geyer, H., Thomas, A., Braun, H., Haenelt, N.,... Shänzer W. (2012). Clenbuterol-regional food contamination a possible source for inadvertent doping in sports. Drug Testing and Analysis, 4, 534-538. doi:101002/dta.1330.

Hemmersbach, P. (2008). History of mass spectrometry at the Olympic Games. Journal of Mass Spectrometry, 43, 839 -853. doi:10.1002/jms.1445.

Instituto Mexicano de Normalización y Certificación, (IMNC) México (2006) NMX-EC-17025:IMNC:2006 “Requisitos generales para la competencia de los laboratorios de ensayo y calibración".

Kickman, A. y Cowan, D. (2009). Subject-based profiling for the detection of testosterone administration in sport. Drug Testing and Analysis, 1, 22-24. doi:10.1002/dta.14.

Kioukia, N., Fragkaki, A., Polyxeni, K., Pelagio, I., Dimopoulou, H., Tsivou, M.,... Georgakopoulos, C. (2014). A synopsis of the adverse analytical and atypical findings between 2005 and 2011 from the Doping Control Laboratory of Athens in Greece. Journal of Analytical Toxicology, 38, 16-23. doi:10.1093/jat/bkt089.

Koehler, K., Braun, H., De Marees, M., Geyer, H., Thevis, M., Mester, J. y Schaenzer, W. (2011). Urinary excretion of exogenous glycerol administration at rest. Drug Testing and Analysis, 3, 877-882. doi:10.1002/dta.355.

Marclay, F., Grata, E., Perrenoud, L. y Saugy, M. (2011). A one-year monitoring of nicotine use in sport: Frontier between potential performance enhancement and addiction issues. Forensic Science International, 323, 73-84. doi:10.1016/j.forsciint.2011.05.026.

Mareck, U., Geyer, H., Opfermann, G., Thevis, M. y Schänzer, W. (2008). Factors influencing the steroid profile in doping control analysis. Journal of Mass Spectrometry, 43, 877-891. doi:10.1002/jms.1457.

Mareck, U., Haenelt, N., Geyer, H., Guddat, S., Kamber, M., Brenneisen, R.,... Schänzer, W. (2009). Temporal indication of cannabis use by means of THC glucuronide determination. Drug Testing and Analysis, 1, 505-510. doi:10.1002/dta.106. 
Möller, I., Wintermeyer, A., Bender, K., Jübner, M., Thomas, A., Krug, O.,... Thevis, M. (2011). Screening for the synthetic cannabinoid JWH-018 and its major metabolites in human doping controls. Drug Testing and Analysis, 3, 609-620. doi:10.1002/dta.158.

Monfort, N., Martínez, L., Bergés, R., Segura, J. y Ventura, R. (2015). Screening method for stimulants in urine by UHPLC-MS/MS: identification of isomeric compounds. Drug Testing and Analysis, 7, 819-830. doi:10.1002/ dta. 1776 .

O’Byrne, P., Kavanagh, P., McNamara, S. y Stokes, S. (2013). Screening of stimulants including designer drugs in urine using a liquid chromatography tandem mass spectrometry system. Journal of Analytical Toxicology, 37, 64-73. doi:10.1093/jat/bks091.

Parr, M. K., Blokland, M. H., Liebetrau, F., Schmidt, A. H., Meijer, T., Stanic, M.,... Sterk, S. S. (2017). Distinction of clenbuterol intake from drug or contaminated food of animal origin in a controlled administration trial The potential of enantiomeric separation for doping control analysis. Food Additives Contaminants: Part A, 34, 525-535. doi:10.1080/19140049.2016.

Ramos, A. (1999). Lucha contra el dopaje como objetivo de salud. Adicciones, 11, 299-310. doi:10.20882/adicciones.11.4.

Saugy, M., Lundby, C. y Robinson, N. (2014). Monitoring of biological markers indicative of doping: the athlete biological passport. British Journal Sports Medicine, 48, 827-832. doi:10.1136/bjsports-2014-093512.

Smith, A. y Stewart, B. (2015). Why the war on drugs in sport will never be won. Harm Reduction Journal, 12, 1-6. doi:10.1186/s12954-015-0087-5.

Sottas, P., Robinson, N., Rabin, O. y Saugy, M. (2011). The athlete biological passport. Clinical Chemistry, 57, 969976. doi:10.1373/clinchem.2011.162271.

Strano, S., Abate, M., Bragano, M. y Botrè, F. (2009). Consumo de sustancias estimulantes y drogas de abuso en el deporte: la experiencia italiana. Adicciones, 21, 239 -242. doi:10.20882/adicciones.21.3.

Thevis, M., Geyer L., Geyer H., Guddat, S., Dvorak, J., Butch, A.,... Schänzer W. (2013). Adverse analytical findings with clenbuterol among U-17 soccer players attributed to food contamination issues. Drug Testing and Analysis, 5, 372-376. doi:10.1002/dta.1471.

Thevis, M., Hemmersbach, P. Geyer, H. y Schänzer, W. (2009). Doping im Behindertensport. Medizinische Klinik, 104, 918-924. doi: 10.1007/s00063-009-1190-8.

Thevis, M., Kuuranne, T., Geyer, H. y Schänzer, W. (2017). Annual banned-substance review; analytical approaches in human sports drug testing. Drug Testing and Analysis, 9, 6-29. doi:10.1002/dta.2139.

Thevis, M., Thomas, A., Beuk, S., Butch, A., Dvorak, J. y Schänzer, W. (2013). Does the analysis of the enantiomeric composition of clenbuterol in human urine ena- ble the differentiation of illicit clenbuterol administration from food contamination in sports drug testing. Rapid Communications in Mass Spectrometry, 27, 507-512. doi:10.1002/rcm.6485.

Thörngren, J., Östervall, F. y Garle, M. (2008). A high-throughput multicomponents screening method for diuretics, masking agents, central nervous system (CNS) stimulants and opiates in human urine by UPLC-MS/MS. Journal of Mass Spectrometry, 43, 980-992. doi:10.1002/ jms.1436.

Velasco-Bejarano, B., Bautista, J., Noguez, M. O., Camacho, E., Rodríguez, M. E. y Rodríguez, L. (2017). Resolution of $R-(-)$ and $S(+)$-enantiomers of clenbuterol in pharmaceutical preparations and black-market products using liquid chromatography-tandem mass spectrometry. Drug Testing and Analysis, 9, 1738-1743. doi:10.1002/ dta.2294.

WADA-AMA, ISL, 2009, 2012, 2015. International Standard for Laboratories (ISL). Recuperado de https://www. wada-ama.org/sites/default/files/resources/files/isl_ june_2016.pdf.

WADA-AMA, PL, 2009, 2010, 2011, 2012, 2013, 2015. The World Anti-doping Code Prohibited List International Standard. Recuperado de https://www.wada-ama.org/en/ resources/science-medicine/prohibited-list-documents.

WADA-AMA, WLS, 2009, 2011, 2012, 2013, 2014, 2015. WADA Laboratory Statistics. Recuperado de https:// www.wada-ama.org/en/resources/laboratories/anti-doping-testing-figures.

WADA-AMA, SCP, 2006. Sample Collection Personnel: Recruitment, Training, Acreditation and Re-Acreditation Guideline. Recuperado de https://www.wada-ama.org/sites/default/files/resources/files/wada_guidelines_sample_ collection_personnel_2014_v1.0_en.pdf .

WADA-AMA, TD-DL, 2010, 2012, 2013, 2014. The World Antidoping Code, WADA Technical Document, Decision Limits for the Confirmatory Quantification of Threshold Substances (DL). Recuperado de https://www.wada-ama.org/sites/ default/files/resources/files/wada-td2017dl-v2-en_0. pdf.

WADA-AMA, TD-EAAS, 2004, 2014. The World Antidoping Code, WADA Technical Document, Endogenous Anabolic Androgenic Steroids Measurement and Reporting (EAAS). Recuperado de https://www.wada-ama.org/sites/default/ files/resources/files/wada-td2016eaas-eaas-measurement-and-reporting-en.pdf.

WADA-AMA, TD-IRMS, 2014. The World Antidoping Code, WADA Technical Document, Detection of synthetic forms of endogenous anabolic androgenics steroids by GC-C-IRMS (IRMS). Recuperado de https://www.wada-ama.org/sites/default/files/resources/files/wada-td2016irms-detection_synthetic_forms_eaas_by_irms-en.pdf.

WADA-AMA, IDCR, 2010, 2013, 2015. The World Antidoping Code, WADA Technical Document, Minimum criteria for chro- 
matographic-Mass spectrometric confirmation of the identity of analytes for doping control purposes (IDCR). Recuperado de https:/ /www.wada-ama.org/sites/default/files/resources/files/td2015idcr_-_eng.pdf.

WADA-AMA, TD-MRPL, 2009, 2010, 2012, 2014, 2015. The World Antidoping Code, WADA Techincal Document, Minimum Required Performance Levels for Detection and Identification of Non-Threshold Substances (MRPL). Recuperado de https://www.wada-ama.org/sites/default/files/resources/files/td2018mrpl_v1_finaleng.pdf.

WADA-AMA, TD-2017NA, 2009, 2010, 2012, 2014, 2015. The World Antidoping Code, WADA Technical Document, Harmonization of analysis and reporting of 19-Norsteroids related to nandrolone (NA). Recuperado de https://www. wada-ama.org/sites/default/files/resources/files/wada-td2017na-en_0.pdf.

WADA-AMA, TD-EPO, 2009, 2013, 2014. The World Antidoping Code, WADA Technical Document, Harmonization of the Method for the Identification of Epoetin Alfa and Beta (EPO) and Darbepoetin Alfa (NESP) by IEF-Double Blotting and Chemiluminescent Detection (EPO). Recuperado de https:// www.wada-ama.org/sites/default/files/resources/ files/WADA-TD2014EPO-v1-Harmonization-of-Analysis-and-Reporting-of-ESAs-by-Electrophoretic-Techniques-EN.pdf.

WADA-AMA, TD-LDOC, 2009. The World Antidoping Code, WADA Technical Document, Laboratory Documentation Packages (LDOC). Recuperado de https://www.wada-ama. org/sites/default/files/resources/files/td2017ldoc_ v2_en.pdf.

WADA-AMA, TD-ICOC, 2009. The World Antidoping Code, WADA Technical Document, Laboratory Internal Chain of Custody (ICOC). Recuperado de https://www.wada-ama. org/sites/default/files/resources/files/WADA_ TD2009LCOC_Laboratory_Internal_Chain_Custody_ EN.pdf.

WADA-AMA, TD-NAND, 2004. The World Antidoping Code, WADA Technical Document, Reporting Norandrosterone Findings (NAND). Recuperado de http:/ / www.wada-ama.org.

World Anti-Doping Agency. Acerca de la WADA-AMA. Recuperado de https://www.wada-ama.org. 